\title{
Methods of using carbon nanotubes as filter media to remove aqueous heavy metals
}

Yuan Tian, Bin Gao, Verónica L. Morales, Lei Wu, Yu Wang, Rafael Muñoz-Carpena, Can Cao1, Qingguo Huang, and Liuyan Yang

NOTICE: this is the author's version of a work that was accepted for publication in Chemical Engineering Journal. Changes resulting from the publishing process, such as peer review, editing, corrections, structural formatting, and other quality control mechanisms may not be reflected in this document. Changes may have been made to this work since it was submitted for publication. A definitive version was subsequently published in Chemical Engineering Journal, [Vol. 210, (2012)] DOI http://dx.doi.org/10.1016/j.cej.2012.09.015 
Methods of using carbon nanotubes as filter media to remove aqueous heavy metals

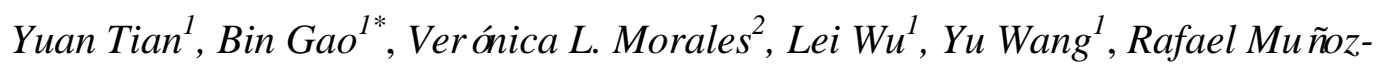
Carpena ${ }^{1}$, Can Cao ${ }^{1}$, Qingguo Huang ${ }^{3}$, and Liuyan Yang ${ }^{4}$

1. Department of Agricultural and Biological Engineering, University of Florida, Gainesville, FL 32611-0570

2. SIMBIOS Centre, University of Abertay, Dundee, DDI U.K.

3. Department of Crop and Soil Sciences, University of Georgia, Griffin, GA 30223

4. State Key Laboratory of Pollution Control and Resource Reuse, School of the Environment, Nanjing University, Nanjing 210093, China

*Corresponding Author, Phone: (352) 392-1864 ext.285, email: bg55@ufl.edu 


\begin{abstract}
Although carbon nanotubes (CNTs) are well known to have a strong affinity to various heavy metals in aqueous solution, little research has been dedicated to exploit their use in fixed-bed water treatment systems (e.g., trickling filters). In this work, batch sorption and fixed-bed experiments were conducted to examine the ability of functionalized multi-walled CNTs as filter media to remove two heavy metal ions $\left(\mathrm{Pb}^{2+}\right.$ and $\mathrm{Cu}^{2+}$ ) from infiltrating water. Batch sorption experiments confirmed the strong sorption affinity of the CNTs for $\mathrm{Pb}^{2+}$ and $\mathrm{Cu}^{2+}$ in both single and dual metal solution systems. In addition, sonication-promoted dispersion of the CNT particles enhanced their heavy metal sorption capacity by $23.9-32.2 \%$. For column experiments, laboratory-scale fixed-bed columns were packed with CNTs and natural quartz sand by three different packing: layered, mixed, and deposited. While all the three packing methods enhanced the fixed-bed filtering efficiency of $\mathrm{Pb}^{2+}$ and $\mathrm{Cu}^{2+}$ from single and dual metal systems, the CNT-deposited packing method was superior. Although the amount of the CNTs added into the fixed-bed columns was only $0.006 \%(\mathrm{w} / \mathrm{w})$ of the sand, they significantly improved the fixed-bed's filtering efficiency of $\mathrm{Pb}^{2+}$ and $\mathrm{Cu}^{2+}$ by $55 \%-75 \%$ and $31 \%$ $57 \%$, respectively. Findings from this study demonstrate that functionalized multi-walled CNTs, together with natural sand, can be used to effectively and safely remove heavy metals from water.
\end{abstract}

Keywords: carbon nanotube, natural sand, fixed-bed, heavy metal, sorption, filtration 


\section{Introduction}

The exceptional properties of nanomaterials, particularly carbon nanotubes (CNT), have revolutionized the electronic and optic industries, energy sectors, and material engineering and manufacturing. Much research on carbon-based nanoparticle in the environment has been devoted to elucidate the physical, chemical, and biological mechanisms that affect their stability, mobility, and toxicity [1]. Relatively less attention has been paid to their potential application for addressing a number of environmental problems $[2,3]$.

The hollow and layered nanostructure of CNTs endow them with a characteristically large surface area and a correspondingly high potential sorption capability for chemical pollutants [4]. Several studies have demonstrated that CNTs, particularly those that are functionalized, have a strong affinity to many common water pollutants, including heavy metals [5] and organic pollutants [6]. It is well accepted that pristine CNTs are insoluble in water [7], therefore oxidative treatments are often used to introduce hydrophilic functionalities to their surfaces in order to facilitate aqueous dispersion. This functionalization procedure is suspected to improve CNTs' ability to remove heavy metals in aqueous phase by increasing their cation exchange capacity as well as promoting attractive electrostatic interactions [8]. As such, functionalized CNTs have been reported to have a superior sorption ability to heavy metal ions than pristine CNTs and have been recommended as a potential adsorbent for the removal of heavy metals in contaminated water [9-11].

A vast proportion of published investigations concerned with the removal of contaminants by CNTs have investigated sorption characteristics and mechanisms 
through batch sorption experiments $[5,12,13]$. To the authors' knowledge, no study has previously examined the feasibility and effectiveness of using CNTs as filter media in fixed-bed settings to remove contaminants from aqueous solutions. A potential deterrent for the novel application of CNTs for water treatment is the concern of nanoparticle elution from the fixed-bed reactors, which could result in secondary contamination of the receiving water bodies.

A recent study by the authors suggested that natural sand media can be used as a good natural filter to remove functionalized CNTs from water [5]. The deposition of functionalized CNTs on natural sand surface is mainly controlled by strong surface interactions that are irreversible despite chemical and hydrodynamic disturbances [12]. This makes it possible to envision the development of a new technology to use sand as a safeguard for functionalized CNTs in a fixed-bed filter to remove contaminants from water. Sand is very a common filter material used in water treatment, but it has relatively poor ability to remove heavy metals from water $[13,14]$. We hypothesize that the combination of sand media with functionalized CNTs would greatly improve the performance of fixed-beds for removing heavy metals from water.

The overarching goal of this work is to assess the best procedures and their respective effectiveness of the CNT-sand filter media for heavy metal removal from water. First, functionalized multi-walled CNTs were incorporated to natural quartz sand media in laboratory fixed-bed columns via three packing methods: 1) layered, where CNTs and sand were packed as separate layers in the columns; 2) mixed, where CNTs and sand were mixed together and subsequently packed in the columns; and 3) deposited, where a well-dispersed CNT suspension was applied to the columns and allowed to 
become deposited onto sand surface via filtration. Second, filtration experiments were conducted with the CNT-sand columns in which the removal efficiency of two heavy metals in aqueous phase $\left(\mathrm{Pb}^{2+}\right.$ and $\left.\mathrm{Cu}^{2+}\right)$ was evaluated. The specific objectives of the work included: 1) determine the effect of sonication-promoted dispersion on sorption capacity of the functionalized multi-walled CNTs to $\mathrm{Pb}^{2+}$ and $\mathrm{Cu}^{2+}$ in single and dual (competing) metal systems; 2) compare removal efficiency of single metal solution $\left(\mathrm{Pb}^{2+}\right.$ or $\mathrm{Cu}^{2+}$ ) across the three packing types of CNT-sand media; and 3) compare removal efficiency of dual metal solution $\left(\mathrm{Pb}^{2+}\right.$ and $\left.\mathrm{Cu}^{2+}\right)$ across the three packing types of CNTsand media.

\section{Materials and methods}

\subsection{CNTs}

Multi-walled CNTs were produced using a chemical vapor deposition method with nickel and magnesium catalysts. Subsequently, these were functionalized by an acid mixture of concentrated sulfuric and nitric acids $(3: 1, \mathrm{v}: \mathrm{v})$ to introduce carboxyl and hydroxyl functional groups to the nanotube surface $[14,15]$. CNTs produced from this method have high purity with negligible metal content [15]. Average diameter and length of the CNTs were around 40 and $400 \mathrm{~nm}$, respectively, as determined with scanning electron microscope (JEOL 6335F, Tokyo, Japan) [16]. Fourier transform infra-red (FTIR) analysis was used to characterize those functional groups. To obtain the observable adsorption spectra, the CNTs were mixed with $\mathrm{KBr}$ to 0.1 wt \% and then pressed into pellets. The spectra were measured using a Bruker Vector 22 FTIR spectrometer (OPUS 2.0 software). 
Part of the functionalized CNTs was used directly in some of the sorption and column experiments without any dispersion promoting treatments and was referred here as undispersed CNTs. A separated dispersed CNT suspension was produced by suspending the functionalized CNTs in deionized (DI) water at a desired concentration and then sonicated with Misonix S3000 ultrasonicator (QSonica, Newtown, CT) for 30 min, and was referred here as dispersed CNTs.

The physiochemical characteristics of the CNTs were measured for: i) Surface area via the Brunauer-Emmett-Teller (BET) nitrogen adsorption method at $77 \mathrm{~K}$ (NOVA 1200 surface area analyzer, Quantachrome Instruments, Boynton Beach, FL), ii) point of zero charge (PZC) via the mass titration method [17], iii) hydrodynamic diameter of dispersed CNTs by dynamic light scattering (ZetaPlus, Brookhaven Instruments Corporation, Holtsville, NY), and iv) electrophoretic mobility (EPM) of dispersed CNTs (ZetaPlus), which was used estimating their zeta potential via Smoluchowski's model. Concentrations of the dispersed CNT suspensions were determined through measuring their spectroscopic light absorption at a wavelength of $255 \mathrm{~nm}$ using an Evolution 60 UV-Vis Spectrophotometer (Thermo Scientific, Waltham, MA). The absorbance spectra and calibration curve of the CNT suspensions at this wavelength can be found in the supplementary data (Figure S1). FTIR analysis was conducted with the post-sorption CNTs (i.e., metal laden CNTs) to examine the functional groups using the method described above. 


\subsection{Sand}

Quartz sand of grain size 0.5-0.6 mm (Standard Sand \& Silica Co., Davenport, FL) was washed sequentially with tap water and DI water to remove loose impurities. Quartz fragmentation by ultrasonication procedure proposed by Johnson et al. [18] was used to create quartz colloids, which were subsequently filtered through a $0.45 \mu \mathrm{m}$ filter. The quartz suspension was then used to measure the sand's EMP and PZC. Trace metal identification and concentration on the sand surface were determined by inductively coupled plasma with optical emission spectrometry (ICP-OES, Optima 2100 DV, PerkinElmer Inc., Waltham, MA), as per EPA protocol 200.7.

\subsection{Heavy metals}

Lead nitrate and copper nitrate were used to prepare stock solutions. Single metal solutions were prepared at concentrations of $10 \mathrm{mg} \mathrm{L}^{-1}$ of $\mathrm{Pb}^{2+}$ or $\mathrm{Cu}^{2+}$. In addition, a dual metal solution containing $10 \mathrm{mg} \mathrm{L}^{-1}$ of $\mathrm{Pb}^{2+}$ and $10 \mathrm{mg} \mathrm{L}^{-1}$ of $\mathrm{Cu}^{2+}$ was prepared. ICP-OES was used to monitor the metal concentration in the solutions. $\mathrm{HCl}$ was used to adjust the $\mathrm{pH}$ of the metal solutions to 5.6.

\subsection{Batch sorption experiment}

Batches of each sorbent (dry CNTs, dispersed CNTs, or 1g of sand) were added to $20 \mathrm{~mL}$ of each metal solution $\left(\mathrm{Pb}^{2+}, \mathrm{Cu}^{2+}\right.$, and $\mathrm{Pb}^{2+}$ and $\left.\mathrm{Cu}^{2+}\right)$ at seven different concentrations to build adsorption isotherms. The resultant CNT dosages were $10 \mathrm{mg}$ in the $20 \mathrm{~mL}$ of the mixture. The mixtures were shaken in a shaker for $12 \mathrm{~h}$ at room temperature, which was determined sufficient time to ensure equilibration $[19,20]$. After 
equilibrium was reached, the suspensions were filtered through $0.1 \mu \mathrm{m}$ membranes, and the initial and equilibrium metal concentrations then measured using the ICP-OES. The mass of sorbed metal was calculated as the difference between initial and equilibrium metal concentration.

\subsection{Fixed-bed column experiment}

Fixed-bed column experiments were used to investigate the removal and transport of heavy metals in CNT-sand media via three different CNT packing methods. Layered, mixed and deposited CNTs were the three ways in which about $10 \mathrm{mg}$ of CNTs were incorporated into $16.8 \mathrm{~g}$ of sand for each CNT-sand packed column. Columns packed with only natural sand were used as the control in the study.

For the layered CNT packing method, natural sand was first wet-packed into an acrylic column (1.5 cm inside diameter $5 \mathrm{~cm}$ height), and undispersed CNTs were evenly placed on the top of the sand packed in the column as a separated layer. Membranes with $50 \mu \mathrm{m}$ pores (Spectra/Mesh, Spectrum Laboratories, Inc.) were used at the column inlet and outlet to distribute the flow. For the mixed CNT packing method, natural sand was thoroughly mixed with the undispersed CNTs and subsequently wet packed into the column. For the deposited CNT packing method, the column was first wet-packed with the sand, and the dispersed CNT suspension was then applied as a pulse (in downward flow direction) using a peristaltic pump set at a steady flow rate of 1 $\mathrm{mL} / \mathrm{min}$. After continuous CNT pulse injection, the influent was switched to DI water for an additional 2 hrs to flush out unretained CNTs. Effluent concentration of CNTs was monitored in discrete samples via UV-Vis Spectrophotometry at a wavelength of $255 \mathrm{~nm}$ 
to determine when $10 \mathrm{mg}$ of CNTs had been deposited in the sand column. Because small amount of CNTs $(\sim 0.06 \% \mathrm{w} / \mathrm{w}$ of CNT/sand $)$ were used in the fixed-bed columns, the bed porosity of all three types of column packing was maintained at 0.40 .

Once packed with sand and CNTs, the columns were subjected to pulses of single and dual metal solutions and the removal efficiency evaluated according to the metal breakthrough from the columns. Prior to metal pulse injection, the columns were flushed with DI water ( $\mathrm{pH}$ 5.6) for more than $1 \mathrm{hr}$ and no CNT was detected in the eluents of any the columns. Metal removal experiments consisted of two stages. At stage one, the single $\left(\mathrm{Pb}^{2+}\right.$ or $\left.\mathrm{Cu}^{2+}\right)$ or dual $\left(\mathrm{Pb}^{2+}\right.$ and $\left.\mathrm{Cu}^{2+}\right)$ metal solutions (as described above) were injected into the top of the column for $2 \mathrm{hrs}$ at a steady flow rate of $1 \mathrm{~mL} / \mathrm{min}$. At stage two, the influent was switched to metal-free DI water for an additional 2 hrs to elute unfiltered metals from the column. Effluent samples were collected discretely with a fraction collector and the metal concentration monitored with the ICP-OES.

\section{Results and discussion}

\subsection{CNT and sand properties}

In dispersion, CNTs were very stable and their concentration maintained unchanged after 24 hrs (Figure S2, supplementary data). All the experiments with dispersed CNTs were finished within 24 hrs to avoid any potential aggregations. The $\mathrm{pH}$ of the dispersed CNT suspension was 5.6. Hydrodynamic diameter of the dispersed CNTs was around $180 \mathrm{~nm}$, and their EPM was recorded at $-2.99 \times 10^{-8} \mathrm{~m}^{2} \mathrm{~V}^{-1} \mathrm{~s}^{-1}$. Mass titration curves (Figure S3, supplementary data) indicated that PZC of the CNTs was 2.4, corroborating their negative charge under the tested experimental conditions. Those surface charges 
may arise from the abundances of acidic functional groups. FTIR spectrum of the CNTs were characterized by four significant bands at wave number 3445 (O-H stretch), 1635 ( $\mathrm{C}=\mathrm{O}$ bond), 1045 (alkene, $\mathrm{C}-\mathrm{O}$ bond), and 1385 (vibrational band of $-\mathrm{NO}_{2}$ ) $\mathrm{cm}^{-1}$ (Figure 1). The first three bands confirmed the presences of carboxyl and hydroxyl functional groups on the CNT surface.

BET nitrogen adsorption measurement revealed a surface area of undispersed CNT powder of $112.7 \mathrm{~m}^{2} \mathrm{~g}^{-1}$, a significantly lower value than previously reported for welldispersed CNTs $\left(>1000 \mathrm{~m}^{2} \mathrm{~g}^{-1}\right)$ [21]. This result suggests that undispersed CNT powders cannot exhibit their 'true' surface area, unless they are well-dispersed in a medium. Thus, it is anticipated that the dispersed CNTs could display higher sorption capacity for metals than the undispersed CNTs based solely on the available surface area for sorption.

The PZC of the sand was 3.0 (Figure S1, supplementary data), indicating that its charge was negative under the tested conditions. Surface element analysis revealed the presence of metal impurities (mainly iron oxyhydroxides) on the sand surface [13], which could potentially serve as adsorption sites for heavy metal ions [17].

\subsection{Sorption ability of the CNTs}

Both dispersed and undispersed CNTs were much more efficient adsorbents of heavy metals than the sand (Figure 2), which is consistent with the literature that functionalized CNTs have strong affiliations to heavy metal ions in aqueous solutions [14, 22-25]. The dispersed CNTs had greater adsorption ability to both aqueous metals in single and dual metal systems than the undispersed CNTs, which probably could be attributed to the lager surface area of the dispersed CNTs. The isotherms also suggest that 
the sonication-promoted dispersion processes could potentially improve the performance of the CNTs in water treatment to remove heavy metal contaminants.

The Langmuir model used to describe the experimental isotherms was: $\frac{1}{q_{e}}=\frac{1}{q_{m}}+\frac{1}{K q_{m} C_{e}}$, where $q_{e}$ is the mass of metals sorbed per mass of sorbent at equilibrium ( $\left.\mathrm{mg} \mathrm{g}^{-1}\right), q_{m}$ the maximum mass of metals sorbed per mass of sorbent as the concentration of metal increases $\left(\mathrm{mg} \mathrm{g}^{-1}\right), K$ is the Langmuir equilibrium constant for mass of metal $\left(\mathrm{L} \mathrm{mg}^{-1}\right)$, and $C_{e}$ the dissolved metal concentration at equilibrium. The model fit the experimental data very well, as indicated by $R^{2}$ values exceeding 0.92 (Table 1).

The $q_{m}$ values of $\mathrm{Pb}^{2+}$ and $\mathrm{Cu}^{2+}$ sorption on the sand were low in both single and dual metal systems (Table 1), indicating that natural sand alone is not an effective sorbent for heavy metals in solution. The single-metal maximum sorption capacity of the CNTs was 74.5 and $92.3 \mathrm{mg} \mathrm{g}^{-1}\left(0.36\right.$ and $\left.0.45 \mathrm{mmol} \mathrm{g}^{-1}\right)$ for $\mathrm{Pb}^{2+}$ and 51.3 and $67.8 \mathrm{mg} \mathrm{g}^{-1}$ $\left(0.81\right.$ and $\left.1.07 \mathrm{mmol} \mathrm{g}^{-1}\right)$ for $\mathrm{Cu}^{2+}$ for undispersed and dispersed CNTs, respectively. The maximum sorption capacity measured for the CNTs in this study is several fold higher than that of the natural sand and is even better than metal sorption maxima reported for some activated carbons [26-28]. The dual-metal maximum sorption capacity for undispersed CNTs for $\mathrm{Pb}^{2+}$ and $\mathrm{Cu}^{2+}$ was 49.3 and $33.0 \mathrm{mg} \mathrm{g}^{-1}\left(0.24\right.$ and $\left.0.52 \mathrm{mmol} \mathrm{g}^{-1}\right)$, while that for dispersed CNTs was 65.0 and $43.6 \mathrm{mg} \mathrm{g}^{-1}\left(0.31\right.$ and $\left.0.69 \mathrm{mmol} \mathrm{g}^{-1}\right)$, respectively. A likely explanation for higher molar sorption capacity of $\mathrm{Cu}^{2+}$ than for $\mathrm{Pb}^{2+}$ is the atomic radii of these ions, which is 70 and $112 \mathrm{pm}$, respectively. Because of a steric over-crowding, larger ionic radius of $\mathrm{Pb}^{2+}$ compared to that of $\mathrm{Cu}^{2+}$ may induce a quick saturation of adsorption sites, resulting in lower molar capacity [29]. 
Compared to the undispersed CNTs, the sorption capacity of the dispersed CNTs increased for 23.9-32.2\% for $\mathrm{Pb}^{2+}$ and $\mathrm{Cu}^{2+}$ in both single and dual metal systems. These data further confirm the importance of CNT dispersal in order to maximize its sorption capacity for water treatment purposes.

It is suggested that the adsorption of heavy metals onto the CNTs are mainly controlled by the strong interactions between the metal ions and hydrophilic surface functional groups, especially carboxyl and hydroxyl groups [30-32]. Comparison of the FTIR spectra of CNTs before and after metal sorption confirmed this mechanism (Figure 1). Compared to the original FTIR spectrum, the adsorption of heavy metals on the CNTs resulted in variations of FTIR peaks at wavenumbers of $1635(\mathrm{C}=\mathrm{O})$ and $1045(\mathrm{C}-\mathrm{O})$

$\mathrm{cm}^{-1}$, which could be attributed to the interactions between metal ions and carboxyl and hydroxyl groups. Previous studies have suggested that positively charged metal ions can form strong complexes with these two functional groups through electrostatic and/or hydrogen-bonding interactions [1,33-35]. A recent study has suggested that metal impurities in the CNTs could play an important role in their sorption of $\mathrm{Pb}^{2+}$ in aqueous solutions [36]. This mechanism might not be a dominant factor to the sorption of the two heavy metals here because high-purity CNTs were used in this study.

\subsection{Single metal removal in the CNT columns}

The evaluation of three packing methods of CNT-sand media was conducted through analyses of effluent breakthrough of dissolved metals in water. Breakthrough curves (BTCs) for single-metal pulse injections were analyzed for filtration and transport as they moved through the fixed-bed sandy columns with and without CNTs as illustrated 
in Figure 3. The BTCs were constructed as plots of the cumulative pore volume of liquid eluted versus the relative concentration $\left(\mathrm{C} / \mathrm{C}_{0}\right)$ of the injected metal in the effluent. For injections of single-metal $\left(\mathrm{Pb}^{2+}\right.$ and $\left.\mathrm{Cu}^{2+}\right)$ pulses, the BTCs corresponding to fixed-beds with deposited CNTs (inverted triangle in Figure 3) had the lowest mass of metal eluted and the most delayed breakthrough elution, indicating that deposited CNT packing method was the most effective way in improving the fixed-bed's heavy metal removal performance. This superior metal sorption efficiency of deposited CNT fixed-beds agrees with the sorption experiment results that indicated that dispersed CNTs were better sorbents than undispersed CNTs, which were used for layered and mixed CNT fixed-beds. Based on the BTC shapes and arrival times, the metal removal efficiency of the fixed-bed columns was ranked in decreasing order as: deposited CNT > mixed CNT > layered CNT $>$ CNT-free sand.

The following three filtration performance parameters were calculated to evaluate the column packing methods against the different combination of metals in solution: removal rate $\left(r_{m}\right)$, breakpoint $\left(b_{p}\right.$, defined as the $\mathrm{BV}$ at which the metal concentration in the effluent reaches 5\% of the initial metal concentration [37]), and recommended bed capacity $\left(q_{r}\right)$ (Table 2). The recommended bed capacity represents the capacity in practice at which the sorbent in the fixed-bed column should be replaced or regenerated, and can be determined as [38, 39]: $q_{r}=\frac{C_{0} \times Q}{m} \int_{t=0}^{t=t_{h}}\left(1-\frac{C}{C_{0}}\right) \mathrm{d} t$, where $Q$ is the flow rate $\left(\mathrm{L} \mathrm{min}^{-1}\right)$, $m$ is mass of the sorbent in the fixed $(\mathrm{g}), t$ is time $(\mathrm{min})$, and $t_{h}$ is time when the metal concentration in the effluent reaches half of that in the influent (i.e., $C / C_{0}=0.5$ ). As a conservative measure, the calculated $q_{r}$ of a sorbent should not exceed its Langmuir capacity, as determined from batch sorption experiment. 
The CNT-free sand columns were able to remove $32.0 \%$ and $12.5 \%$ of the inputted single-metal $\mathrm{Pb}^{2+}$ and $\mathrm{Cu}^{2+}$, respectively. In contrast, the CNT-sand columns improved the metal removal performance of the columns at $r_{m}$ values of up to $75.0 \%$ for $\mathrm{Pb}^{2+}$ and $56.9 \%$ for $\mathrm{Cu}^{2+}$ for the best performing CNT packing method (deposited), and for $55.3 \%$ for $\mathrm{Pb}^{2+}$ and $31.4 \%$ for $\mathrm{Cu}^{2+}$ for the worst performing CNT packing method (layered). Whereas the range of removal efficiency varied for the three types of CNT packing, the $r_{m}$ of all CNT-sand columns in all cases exceeded that for the CNT-free sand columns. The breakpoints $\left(b_{p}\right)$ of the single-metal pulses in the CNT-sand columns were also much longer than those in the CNT-free sand columns. The most delayed $b_{p}$ was observed for deposited CNT columns, which was recorded at 9.5 min for $\mathrm{Pb}^{2+}$ and $5.2 \min$ for $\mathrm{Cu}^{2+}$, indicating that metal elution in these CNT-enabled columns was delayed by more than twice the time metals were eluted from CNT-free sand columns (Table 2).

The recommended bed capacities $\left(q_{r}\right)$ were the lowest for CNT-free sand columns ( $q_{r}$ of $0.016 \mathrm{mg} \mathrm{g}^{-1}$ for $\mathrm{Pb}^{2+}$ and $0.011 \mathrm{mg} \mathrm{g}^{-1}$ for $\mathrm{Cu}^{2+}$, in Table 2), indicating that sand alone is not an effective heavy metal removal system. The $q_{r}$ values for CNTs in all the CNT-sand columns were three to four orders of magnitude higher than that for sand, with deposited CNTs at the highest capacity and layered CNTs at the lowest (Table 2). These trends are consistent with the findings of individual material batch sorption experiments. The $q_{r}$ values for deposited CNTs in CNT-sand columns (Table 2) corresponded to $87 \%$ and $63 \%$ of their measured Langmuir maximum sorption capacity $q_{m}$ (Table 1$)$ for $\mathrm{Pb}^{2+}$ and $\mathrm{Cu}^{2+}$, respectively. The apparent reduced capacity of the metal sorption for the same quantity of CNTs when added to a fixed-bed system is likely due to the shorter residence time between metal ions and the CNTs when flow of the metal solution was passed 
through the column, compared to the longer contact time between the metals and the sorbents in batch tests.

\subsection{Dual metal removal in the CNT columns}

The three types of CNT-sand columns were also superior at removing $\mathrm{Pb}^{2+}$ and $\mathrm{Cu}^{2+}$ simultaneously from the solution than the CNT-free sand columns (Figure 4). Similar to the single-metal experiments, the metal breakthrough in the CNT-sand columns were lower in mass and more delayed than that in the CNT-free sand columns, where deposited-CNT method was ranked with the best filtration performance. These results are in agreement with the sorption and single-metal removal experiments for dispersed CNT sorption efficiency of metals.

The presence of competing metal ions in the solution for the sorption sites reduced the removal rate, the breakpoint, and the recommended bed capacity in all the columns for the removal of the individual metals (Table 2). It is known that both $\mathrm{Pb}^{2+}$ and $\mathrm{Cu}^{2+}$ cations react strongly with the carboxyl and the hydroxyl groups of the CNT surfaces [39, 40] and competition for these sites on the CNTs is expected. As a result, the removal rates of the two heavy metal ions in the dual metal system in the three CNT-sand columns ranged from $50.2 \%$ to $63.7 \%$ for $\mathrm{Pb}^{2+}$ and $23.6 \%-39.9 \%$ for $\mathrm{Cu}^{2+}$. Although the molar concentration of $\mathrm{Cu}^{2+}$ in the dual metal solution was much higher than that of $\mathrm{Pb}$, the competition only reduced about $9-14 \%$ of $\mathrm{Pb}^{2+}$ removal in the three CNT-sand columns. On the other hand, the presence of $\mathrm{Pb}^{2+}$ reduced about $25-30 \%$ of the $\mathrm{Cu}$ removal. These results suggest that the functionalized $\mathrm{CNT}$ s have a higher affinity to $\mathrm{Pb}^{2+}$ than to $\mathrm{Cu}^{2+}$ ions in aqueous solutions. Similar trend was observed in previous studies of heavy metal 
sorption onto CNTs and it has demonstrated that $\mathrm{Pb}^{2+}$ has a much stronger affinity to the surface function groups on CNTs than $\mathrm{Cu}^{2+}$ in aqueous solutions [31, 35, 39].

\section{Conclusions}

Laboratory experimental results indicated that functionalized multi-walled CNTs had strong sorption ability to aqueous $\mathrm{Pb}^{2+}$ and $\mathrm{Cu}^{2+}$. Dispersion of the CNT particles enhanced their sorption ability to the heavy metals. As a result, the CNT-sand columns packed with CNT deposited method showed the best effect to remove the heavy metal from aqueous solutions. All the three CNT-sand packing methods, however, were effective and safe ways use the CNTs as filter media to remove heavy metal contaminants from water. These results suggest that, the high metal sorption affinity of CNTs along with the high porosity of natural sand could be exploited jointly in a single filter to effectively remove multiple types of heavy metals from water.

Although CNTs have been referred as potentially promising sorbents for heavy metal removal in the literature; however, little research effort has been made to apply them in fix-bed setting to purify water. As the first of this kind of study, findings from this work may inform the development of innovative and high efficiency CNT-based filters for various environmental applications, especially for the treatment of heavy metal contaminated water. With recent capacity scale-ups driving prices down $(\$ 25-38$ per $\mathrm{kg})$ [41], CNTs can make an important contribution to wastewater treatment.

\section{Acknowledgements}

This work was partially supported by the USDA through grant 2009-65102-05847 
and the NSF through grant CBET-1054405. The authors also thank the anonymous reviewers for their helpful suggestions. 


\section{References:}

[1] P. Wang, Q.H. Shi, H.J. Liang, D.W. Steuerman, G.D. Stucky, A.A. Keller, Enhanced Environmental Mobility of Carbon Nanotubes in the Presence of Humic Acid and Their Removal from Aqueous Solution, Small 4 (2008) 2166-2170.

[2] H.F. Lecoanet, M.R. Wiesner, Velocity effects on fullerene and oxide nanoparticle deposition in porous media, Environ. Sci. Technol. 38 (2004) 4377-4382.

[3] Y.A. Tian, B. Gao, C. Silvera-Batista, K.J. Ziegler, Transport of engineered nanoparticles in saturated porous media, J. Nanopart. Res. 12 (2010) 2371-2380.

[4] E.J. Petersen, L.W. Zhang, N.T. Mattison, D.M. O'Carroll, A.J. Whelton, N. Uddin, T. Nguyen, Q.G. Huang, T.B. Henry, R.D. Holbrook, K.L. Chen, Potential Release Pathways, Environmental Fate, And Ecological Risks of Carbon Nanotubes, Environ. Sci. Technol. 45 (2011) 9837-9856.

[5] X.M. Ren, C.L. Chen, M. Nagatsu, X.K. Wang, Carbon nanotubes as adsorbents in environmental pollution management: A review, Chem. Eng. J. 170 (2011) 395-410.

[6] Y.T. Ong, A.L. Ahmad, S.H.S. Zein, S.H. Tan, A Review on Carbon Nanotubes in an Environmental Protection and Green Engineering Perspective, Braz. J. Chem. Eng. 27 (2010) 227-242.

[7] N. Solovitch, J. Labille, J. Rose, P. Chaurand, D. Borschneck, M.R. Wiesner, J.Y. Bottero, Concurrent Aggregation and Deposition of TiO2 Nanoparticles in a Sandy Porous Media, Environ. Sci. Technol. 44 (2010) 4897-4902.

[8] A. Stafiej, K. Pyrzynska, Adsorption of heavy metal ions with carbon nanotubes, Sep. Purif. Technol. 58 (2007) 49-52. 
[9] D. Zhang, B. Pan, H. Zhang, P. Ning, B.S. Xing, Contribution of Different Sulfamethoxazole Species to Their Overall Adsorption on Functionalized Carbon Nanotubes, Environ. Sci. Technol. 44 (2010) 3806-3811.

[10] Y. Tian, B. Gao, K.J. Ziegler, High mobility of SDBS-dispersed single-walled carbon nanotubes in saturated and unsaturated porous media, J. Hazard. Mater. 186 (2011) $1766-1772$.

[11] M.A. Atieh, O.Y. Bakather, B. Al-Tawbini, A.A. Bukhari, F.A. Abuilaiwi, M.B. Fettouhi, Effect of carboxylic functional group functionalized on carbon nanotubes surface on the removal of lead from water, Bioinorg. Chem. Appl. (2010) 603978.

[12] B. Tawabini, S. Al-Khaldi, M. Atieh, M. Khaled, Removal of mercury from water by multi-walled carbon nanotubes, Water Sci. Technol. 61 (2010) 591-598.

[13] M. Rahbari, A.S. Goharrizi, Adsorption of Lead(II) from Water by Carbon Nanotubes: Equilibrium, Kinetics, and Thermodynamics, Water Environ. Res. 81 (2009) 598-607.

[14] Y. Tian, B. Gao, Y. Wang, V.L. Morales, R.M. Carpena, Q. Huang, L. Yang, Deposition and transport of functionalized carbon nanotubes in water-saturated sand columns, J. Hazard. Mater. 213-214 (2012) 265-272.

[15] E.J. Petersen, Q. Huang, W.J. Weber, Jr., Ecological uptake and depuration of carbon nanotubes by Lumbriculus variegatus, Environ. Health Persp 116 (2008) 496-500. [16] Y. Tian, B. Gao, L. Wu, R. Muñoz-Carpena, Q. Huang, Effect of solution chemistry on multi-walled carbon nanotube deposition and mobilization in clean porous media, J. Hazard. Mater. (2012). 
[17] H.M. Sun, B. Gao, Y.A. Tian, X.Q. Yin, C.R. Yu, Y.Q. Wang, L.N.Q. Ma, Kaolinite and lead in saturated porous media: facilitated and impeded transport, J. Environ. Engasce. 136 (2010) 1305-1308.

[18] L. Diels, P.H. Spaans, S. Van Roy, L. Hooyberghs, A. Ryngaert, H. Wouters, E. Walter, J. Winters, L. Macaskie, J. Finlay, B. Pernfuss, H. Woebking, T. Pumpel, M. Tsezos, Heavy metals removal by sand filters inoculated with metal sorbing and precipitating bacteria, Hydrometallurgy 71 (2003) 235-241.

[19] L. Zhang, E.J. Petersen, Q. Huang, Phase Distribution of (14)C-Labeled Multiwalled Carbon Nanotubes in Aqueous Systems Containing Model Solids: Peat, Environ. Sci. Technol. 45 (2011) 1356-1362.

[20] J.S. Noh, J.A. Schwarz, Effect of Hno3 Treatment on the Surface-Acidity of Activated Carbons, Carbon 28 (1990) 675-682.

[21] P.R. Johnson, N. Sun, M. Elimelech, Colloid transport in geochemically heterogeneous porous media: Modeling and measurements, Environ. Sci. Technol. 30 (1996) 3284-3293.

[22] T. Hiraoka, A. Izadi-Najafabadi, T. Yamada, D.N. Futaba, S. Yasuda, O. Tanaike, H. Hatori, M. Yumura, S. Iijima, K. Hata, Compact and Light Supercapacitor Electrodes from a Surface-Only Solid by Opened Carbon Nanotubes with 2200 m(2) g(-1) Surface Area, Adv. Funct. Mater. 20 (2010) 422-428.

[23] M. Mohapatra, A comparative study on $\mathrm{Pb}$ (II), Cd (II), Cu (II), Co (II) adsorption from single and binary aqueous solutions on additive assisted nano-structured goethite, Int. J. Eng. Sci. 2 (2010). 
[24] H.J. Wang, A.L. Zhou, F. Peng, H. Yu, L.F. Chen, Adsorption characteristic of acidified carbon nanotubes for heavy metal $\mathrm{Pb}$ (II) in aqueous solution, Mater. Sci. Eng. A-Struct. Mater. Prop. Microstruct. Process. 466 (2007) 201-206.

[25] X.J. Peng, J.J. Jia, Z.K. Luan, Oxidized carbon nanotubes for simultaneous removal of endrin and $\mathrm{Cd}(\mathrm{II})$ from water and their separation from water, J. Chem. Technol. Biot. 84 (2009) 275-278.

[26] H.J. Wang, A.L. Zhou, F. Peng, H. Yu, J. Yang, Mechanism study on adsorption of acidified multiwalled carbon nanotubes to Pb(II), J. Colloid Interf. Sci. 316 (2007) 277283.

[27] R.A. Doong, L.F. Chiang, Coupled removal of organic compounds and heavy metals by titanate/carbon nanotube composites, Water Sci. Technol. 58 (2008) 1985-1992.

[28] Y.-H. Li, J. Ding, Z. Luan, Z. Di, Y. Zhu, C. Xu, D. Wu, B. Wei, Competitive adsorption of $\mathrm{Pb} 2+, \mathrm{Cu} 2+$ and $\mathrm{Cd} 2+$ ions from aqueous solutions by multiwalled carbon nanotubes, Carbon 41 (2003) 2787-2792.

[29] X.D. Cao, L. Ma, B. Gao, W. Harris, Dairy-manure derived biochar effectively sorbs lead and atrazine, Environ. Sci. Technol. 43 (2009) 3285-3291.

[30] M. Inyang, B. Gao, W. Ding, P. Pullammanappallil, A.R. Zimmerman, X. Cao, Enhanced lead sorption by biochar derived from anaerobically digested sugarcane bagasse, Separ. Sci. Technol. 46 (2011) 1950-1956.

[31] Y.H. Li, J. Ding, Z.K. Luan, Z.C. Di, Y.F. Zhu, C.L. Xu, D.H. Wu, B.Q. Wei, Competitive adsorption of $\mathrm{Pb} 2+, \mathrm{Cu} 2+$ and $\mathrm{Cd} 2+$ ions from aqueous solutions by multiwalled carbon nanotubes, Carbon 41 (2003) 2787-2792. 
[32] J.C. Moreno-Pirajan, R. Gomez-Cruz, V.S. Garcia-Cuello, L. Giraldo, Binary system $\mathrm{Cu}(\mathrm{II}) / \mathrm{Pb}$ (II) adsorption on activated carbon obtained by pyrolysis of cow bone study, $\mathrm{J}$. Anal. Appl. Pyrol. 89 (2010) 122-128.

[33] G.D. Vukovic, A.D. Marinkovic, M. Colic, M.D. Ristic, R. Aleksic, A.A. PericGrujic, P.S. Uskokovic, Removal of cadmium from aqueous solutions by oxidized and ethylenediamine-functionalized multi-walled carbon nanotubes, Chem. Eng. J. 157 (2010) 238-248.

[34] K. Pillay, E.M. Cukrowska, N.J. Coville, Multi-walled carbon nanotubes as adsorbents for the removal of parts per billion levels of hexavalent chromium from aqueous solution, J. Hazard. Mater. 166 (2009) 1067-1075.

[35] G.P. Rao, C. Lu, F. Su, Sorption of divalent metal ions from aqueous solution by carbon nanotubes: A review, Sep. Purif. Technol. 58 (2007) 224-231.

[36] X.L. Tian, S. Zhou, Z.Y. Zhang, X.A. He, M.J. Yu, D.H. Lin, Metal Impurities Dominate the Sorption of a Commercially Available Carbon Nanotube for $\mathrm{Pb}(\mathrm{II})$ from Water, Environ. Sci. Technol. 44 (2010) 8144-8149.

[37] R.H. Tredgold, An Introduction to Ultrathin Organic Films - from LangmuirBlodgett to Self-Assembly - Ulman,A, Nature 354 (1991) 120-120.

[38] B. Saha, S. Das, J. Saikia, G. Das, Preferential and Enhanced Adsorption of Different Dyes on Iron Oxide Nanoparticles: A Comparative Study, J. Phys. Chem. C 115 (2011) 8024-8033.

[39] F.W. Sousa, A.G. Oliveira, J.P. Ribeiro, M.F. Rosa, D. Keukeleire, R.F. Nascimento, Green coconut shells applied as adsorbent for removal of toxic metal ions using fixed-bed column technology, J. Environ. Manage. 91 (2010) 1634-1640. 
[40] S. Yu, G.M. Chow, Carboxyl group (-CO2H) functionalized ferrimagnetic iron oxide nanoparticles for potential bio-applications, J. Mater. Chem. 14 (2004) 2781-2786. [41] A.E. Agboola, R.W. Pike, T.A. Hertwig, H.H. Lou, Conceptual design of carbon nanotube processes, Clean Technologies and Environmental Policy 9 (2007) 289-311. 
Tables

Table 1. Summary of the best-fit Langmuir model parameters.

\begin{tabular}{|c|c|c|c|c|c|c|}
\hline \multirow[b]{2}{*}{ Adsorbent } & \multicolumn{3}{|c|}{$\mathbf{P b}^{2+}$} & \multicolumn{3}{|c|}{$\mathrm{Cu}^{2+}$} \\
\hline & $\begin{array}{c}\mathbf{q}_{\mathrm{m}} \\
\left(\mathrm{mg} \mathrm{g}^{-1}\right) /\left(\mathrm{mmol} \mathrm{g}^{-1}\right)\end{array}$ & $\begin{array}{c}\mathrm{K} \\
\left(\mathbf{L} \mathbf{~ m g}^{-1}\right)\end{array}$ & $\mathbf{R}^{2}$ & $\begin{array}{c}\mathbf{q}_{\mathrm{m}} \\
\left(\mathrm{mg} \mathrm{g}^{-1}\right) /\left(\mathbf{m m o l} \mathbf{g}^{-1}\right)\end{array}$ & $\begin{array}{c}\mathrm{K} \\
\left(\mathbf{L} \mathbf{~ m g}^{-1}\right)\end{array}$ & $\mathbf{R}^{2}$ \\
\hline \multicolumn{7}{|l|}{ Single-metal } \\
\hline Sand & $0.027 / 1.31 \mathrm{E}-04$ & 0.28 & 0.98 & $0.015 / 2.32 \mathrm{E}-04$ & 0.04 & 0.92 \\
\hline Undispersed CNT & $74.5 / 0.36$ & 0.33 & 1.00 & $51.3 / 0.81$ & 0.19 & 0.99 \\
\hline Dispersed CNT & $92.3 / 0.45$ & 0.37 & 0.99 & $67.8 / 1.07$ & 0.31 & 1.00 \\
\hline \multicolumn{7}{|l|}{ Dual-metal } \\
\hline Sand & $0.018 / 8.83 \mathrm{E}-05$ & 0.40 & 0.99 & $0.006 / 9.75 \mathrm{E}-05$ & 0.09 & 0.98 \\
\hline Undispersed CNT & $49.3 / 0.24$ & 0.20 & 1.00 & $33.0 / 0.52$ & 0.07 & 1.00 \\
\hline Dispersed CNT & $65.0 / 0.31$ & 0.21 & 0.98 & $43.6 / 0.69$ & 0.13 & 0.99 \\
\hline
\end{tabular}


Table 2. Summary of fixed-bed column experimental results.

\begin{tabular}{|c|c|c|c|c|c|c|}
\hline \multirow[b]{2}{*}{ Sand column } & \multicolumn{3}{|c|}{$\mathbf{P b}^{2+}$} & \multicolumn{3}{|r|}{$\mathrm{Cu}^{2+}$} \\
\hline & $\begin{array}{c}\mathbf{r}_{\mathrm{m}} \\
(\%)\end{array}$ & $\begin{array}{c}\mathbf{b}_{\mathbf{p}} \\
(\mathrm{min})\end{array}$ & $\begin{array}{c}\mathbf{q}_{\mathrm{r}} \\
\left(\mathrm{mg} \mathrm{g}^{-1}\right) /\left(\mathrm{mmol} \mathrm{g}^{-1}\right)\end{array}$ & $\begin{array}{l}\mathbf{r}_{\mathrm{m}} \\
(\%)\end{array}$ & $\begin{array}{c}\mathbf{b}_{\mathbf{p}} \\
(\min )\end{array}$ & $\begin{array}{c}\mathrm{q}_{\mathrm{r}} \\
\left(\mathrm{mg} \mathrm{g}^{-1}\right) /\left(\mathbf{m m o l ~ g}^{-1}\right)\end{array}$ \\
\hline \multicolumn{7}{|l|}{ Single-metal } \\
\hline CNT-free & 32.0 & 4.4 & $0.016 / 7.72 \mathrm{E}-05$ & 12.5 & 1.2 & $0.006 / 9.44 \mathrm{E}-05$ \\
\hline Layered CNT & 55.3 & 6.0 & $25.9 / 0.13$ & 31.4 & 3.1 & $12.6 / 0.20$ \\
\hline Mixed CNT & 65.9 & 7.3 & $44.3 / 0.21$ & 41.6 & 3.3 & $21.1 / 0.33$ \\
\hline Deposited CNT & 75.0 & 9.5 & $80.1 / 0.39$ & 56.9 & 5.2 & $42.6 / 0.67$ \\
\hline \multicolumn{7}{|l|}{ Dual-metal } \\
\hline CNT-free & 23.1 & 2.4 & $0.011 / 5.31 \mathrm{E}-05$ & 5.4 & 1.2 & $0.006 / 9.44 \mathrm{E}-05$ \\
\hline Layered CNT & 50.2 & 5.4 & $25.3 / 0.12$ & 23.6 & 2.2 & $15.3 / 0.24$ \\
\hline Mixed CNT & 56.7 & 6.7 & $29.9 / 0.14$ & 30.1 & 3.1 & $18.0 / 0.28$ \\
\hline Deposited CNT & 63.7 & 9.5 & $40.9 / 0.20$ & 39.9 & 4.5 & $21.2 / 0.33$ \\
\hline
\end{tabular}




\section{Figure Captions}

Figure 1. FTIR spectra (average of 32 scans) of CNTs before and after heavy metal adsorption.

Figure 2. Sorption isotherms of $\mathrm{Pb}^{2+}$ and $\mathrm{Cu}^{2+}$ onto sand, and dispersed and undispersed CNTs in single and dual metal solutions.

Figure 3. Transport of $\mathrm{Pb}^{2+}$ and $\mathrm{Cu}^{2+}$ in single metal solutions through different types of fixed-bed columns.

Figure 4. Transport of $\mathrm{Pb}^{2+}$ and $\mathrm{Cu}^{2+}$ in dual metal solutions through different types of fixed-bed columns. 
Figure 1

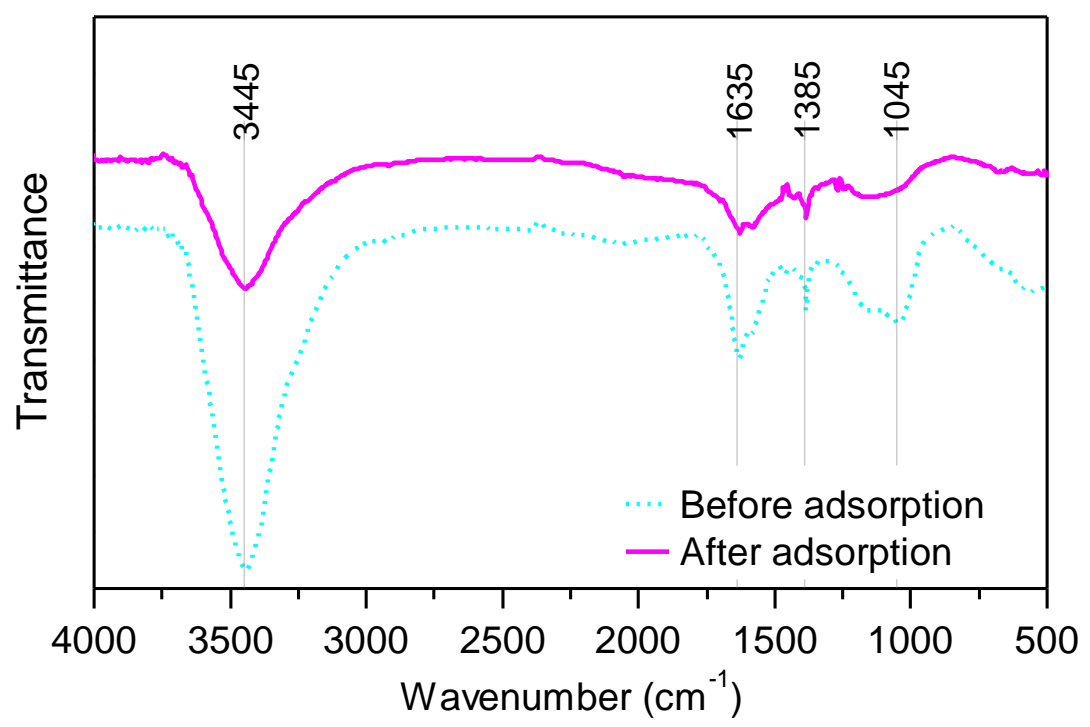


Figure 2
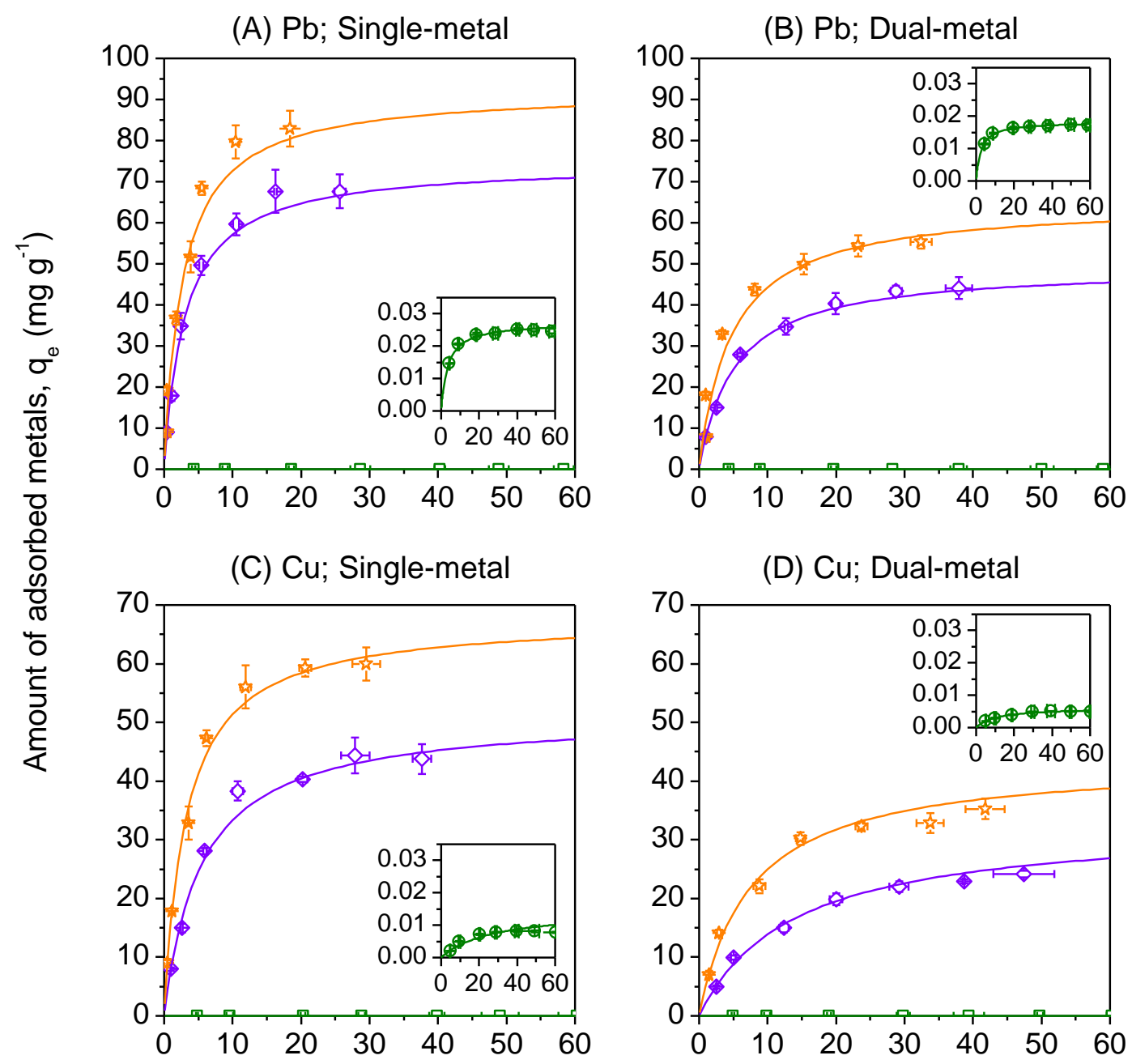

Equilibrium solution concentration, $\mathrm{C}_{\mathrm{e}}\left(\mathrm{mg} \mathrm{L}^{-1}\right)$

$\square$ Sand $\diamond$ Undispersed CNT is Dispersed CNT 


\section{Figure 3}

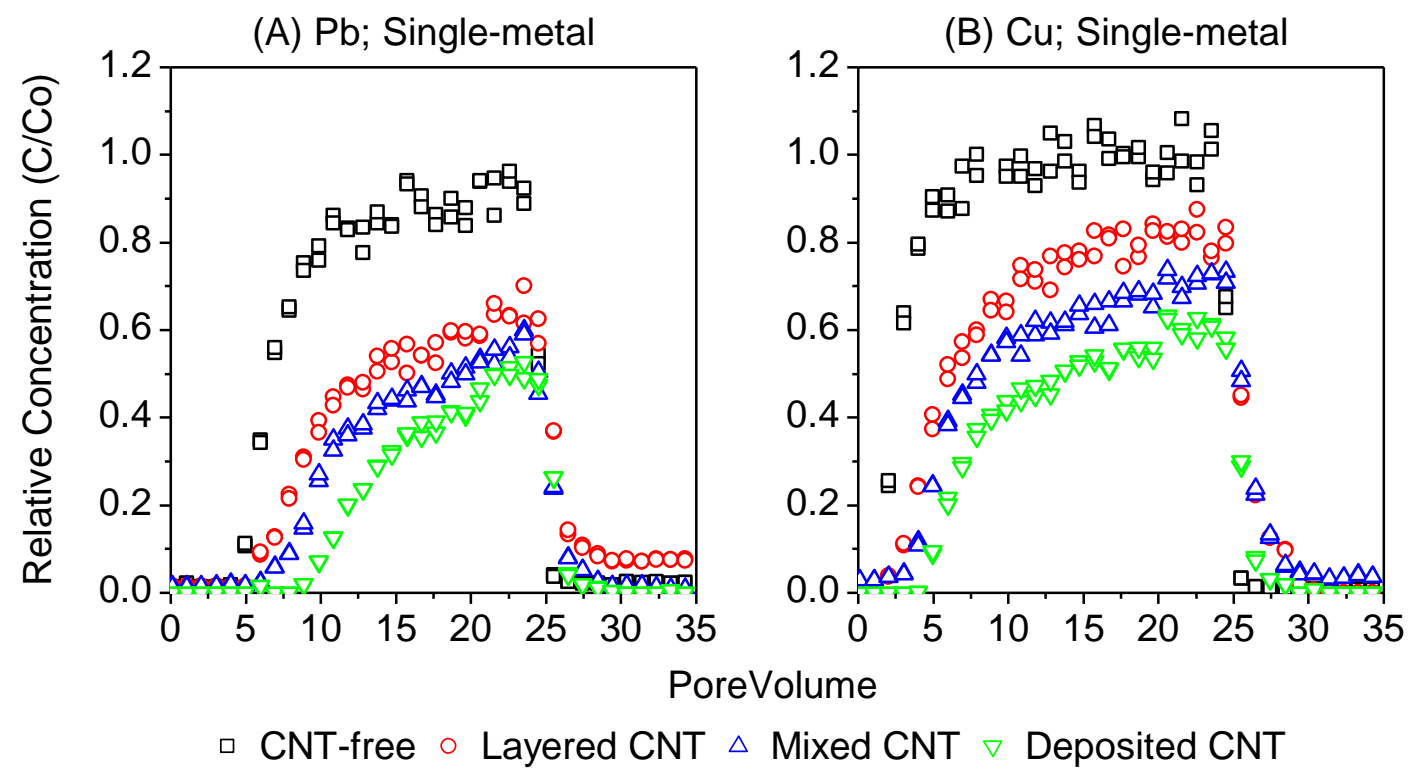




\section{Figure 4}
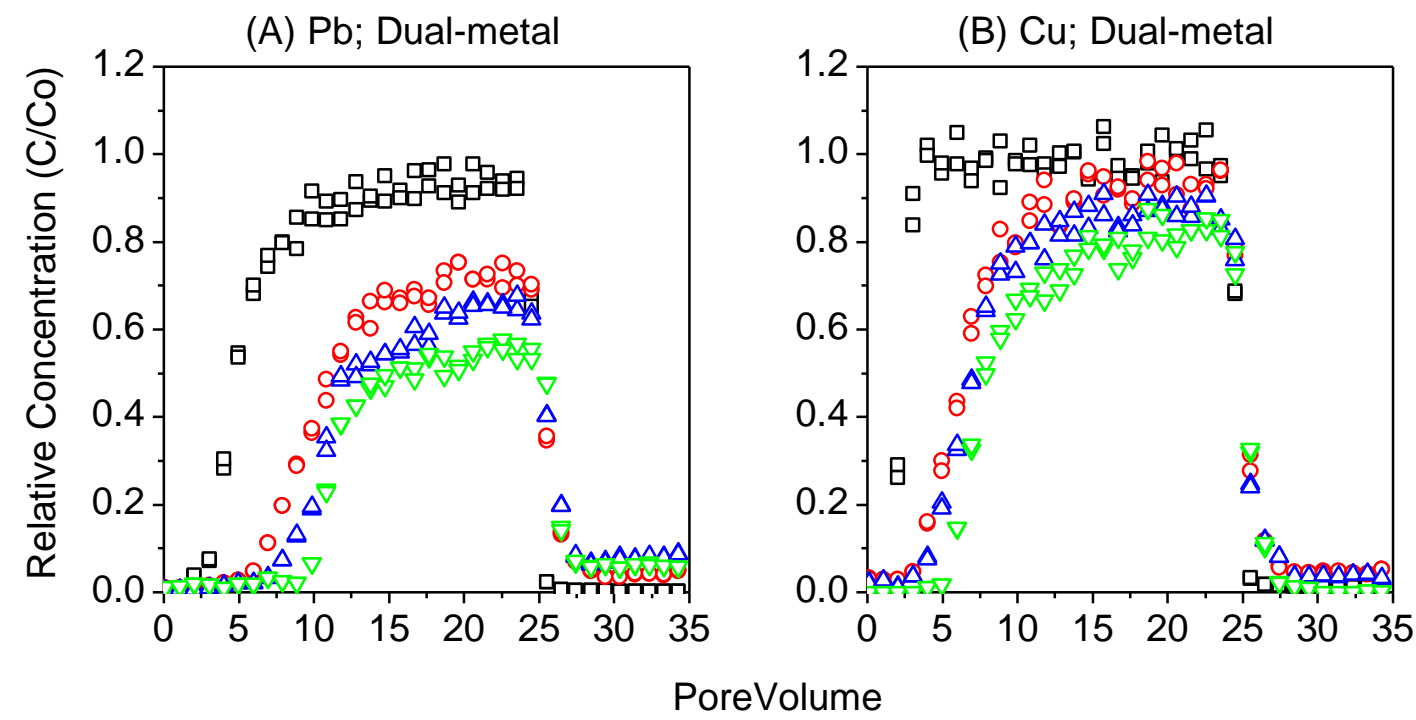

$\checkmark$ CNT-free $\circ$ Layered CNT $\triangle$ Mixed CNT $\nabla$ Deposited CNT 